\title{
SUBEXPONENTIAL ASYMPTOTICS FOR STOCHASTIC PROCESSES: EXTREMAL BEHAVIOR, STATIONARY DISTRIBUTIONS AND FIRST PASSAGE PROBABILITIES
}

\author{
By Søren Asmussen \\ University of Lund
}

\begin{abstract}
Consider a reflected random walk $W_{n+1}=\left(W_{n}+X_{n}\right)^{+}$, where $X_{0}$, $X_{1}, \ldots$ are i.i.d. with negative mean and subexponential with common distribution $F$. It is shown that the probability that the maximum within a regenerative cycle with mean $\mu$ exceeds $x$ is approximately $\mu \bar{F}(x)$ as $x \rightarrow \infty$, and thereby that $\max \left(W_{0}, \ldots, W_{n}\right)$ has the same asymptotics as $\max \left(X_{0}, \ldots, X_{n}\right)$ as $n \rightarrow \infty$. In particular, the extremal index is shown to be $\theta=0$, and the point process of exceedances of a large level is studied. The analysis extends to reflected Lévy processes in continuous time, say, stable processes. Similar results are obtained for a storage process with release rate $r(x)$ at level $x$ and subexponential jumps (here the extremal index may be any value in $[0, \infty]$ ); also the tail of the stationary distribution is found. For a risk process with premium rate $r(x)$ at level $x$ and subexponential claims, the asymptotic form of the infinite-horizon ruin probability is determined. It is also shown by example $[r(x)=a+b x$ and claims with a tail which is either regularly varying, Weibull- or lognormal-like] that this leads to approximations for finite-horizon ruin probabilities. Typically, the conditional distribution of the ruin time given eventual ruin is asymptotically exponential when properly normalized.
\end{abstract}

1. Introduction. This paper deals with reflected random walks, storage processes and risk processes under the assumption that the jumps in the opposite direction of the drift are heavy-tailed (for the precise definition, see Section 1.3). For a reflected random walk or a storage process, we determine the extremal behavior, in particular the asymptotic form of the distribution of the maximum up to time $T$ as $T \rightarrow \infty$. For the storage process, we in addition find the tail of the stationary distribution (which is known for the reflected random walk). By duality, this result gives the asymptotic form of the infinite-horizon ruin probability for the risk process, which turns out to give also the finite-time ruin probabilities or, equivalently, the asymptotic distribution of the ruin time.

1.1. Reflected random walks. Consider a reflected random walk $\left\{W_{n}\right\}_{n=0,1, \ldots}$ (Lindley process) given by the recursion $W_{n+1}=\left(W_{n}+X_{n}\right)^{+}$,

Received October 1996; revised July 1997.

AMS 1991 subject classifications. 60G70,60K30, 60K25.

Key words and phrases. Cycle maximum, extremal index, extreme values, Frechet distribution, Gumbel distribution, interest force, level crossings, maximum domain of attraction, overshoot distribution, random walk, rare event, regular variation, ruin probability, stable process, storage process, subexponential distribution. 
where $X_{0}, X_{1}, \ldots$ are i.i.d. with common distribution $F$ with mean $-m<0$. We are interested in the extreme value behavior of $\left\{W_{n}\right\}$, that is, we want to exhibit constants $a_{n}, b_{n}$ such that

$$
a_{n}\left(M_{n}-b_{n}\right) \rightarrow_{\mathscr{D}} H
$$

for some distribution $H$ where $M_{n}=\max _{k=0, \ldots, n} W_{k}$. More generally, we seek conditions on a real sequence $\left\{u_{n}\right\}$ for $\mathbb{P}\left(M_{n} \leq u_{n}\right)$ to have a limit and we want to study the properties of the random set $\left\{k=0, \ldots, n: W_{k}>u_{n}\right\}$.

It is well known that $\left\{W_{n}\right\}$ is Harris ergodic and hence strongly mixing. Thus, by classical extreme value theory ([26]) and the fact that the stationary distribution $\pi$ has unbounded support, it follows that, up to trivial scaling transformations, only two types of limits in (1.1) can occur: either $H$ is the Frechet distribution $H(x)=\Phi_{\alpha}(x)=\exp \left\{-x^{-\alpha}\right\}, x \geq 0$, for some $\alpha>0$, or $H$ is the Gumbel distribution $H(x)=\Lambda(x)=\exp \left\{-e^{-x}\right\},-\infty<x<\infty$. When $F$ is light-tailed such that exponential moments $\mathbb{E} e^{s X}$ exist, the solution of the problem (1.1) has long been shown ([22]): (1.1) holds with $H=\Lambda$ and $\alpha_{n}=$ $O(1), b_{n}=O(\log n)$. Our contribution here is to deal with the heavy-tailed case where $\mathbb{E} e^{s X}=\infty$ for all $s>0$.

We will see that the behavior in the heavy-tailed case is intrinsically different. In the light-tailed case, $M_{n}$ behaves asymptotically like the maximum of $\theta n$ i.i.d. copies of a random variable $W_{\infty}$ with distribution $\pi$ (see [22] and [28]). Here $\theta>0$ is the extremal index (a precise definition is given below). In contrast, in the heavy-tailed case, $\theta=0$. This situation is somewhat uncommon in extreme value theory and comparatively less general theory has been developed than when $\theta>0$ (cf. [26], pages 71-72). The extremal behavior of $W_{n}$ is indeed like that of an i.i.d. sequence, but the governing distribution is $F$, not $\pi$. More precisely, we will show that $M_{n}$ is close to $\max _{k=0, \ldots, n} X_{k}$. This is in accordance with the common folklore that in the heavy-tailed case, large values occur as a consequence of one big jump; for further results in this vein, see, for example, [13], [1], [5, 6] and [4].

The extreme value results are obtained via the regenerative structure of $\left\{W_{n}\right\}$ w.r.t. the renewal process formed by the visits to 0 . In fact ([10], [22], [28]), the problem of establishing (1.1) is essentially equivalent to finding the asymptotic form of the tail of the cycle maximum. This is carried out in Section 2.1; the extreme value results (incorporating also limit results for the point process of exceedances) themselves are then derived in Sections 2.2-2.3. One crucial ingredient of the proof is the availability of the asymptotic form of the tail of the stationary distribution $\pi$ or, equivalently, the random walk maximum ([16]):

$$
\bar{\pi}(x)=\mathbb{P}(M>x)=\mathbb{P}(\tau(x)<\infty) \approx \frac{1}{m} \int_{x}^{\infty} \bar{F}(y) d y .
$$

Here and in the following, $\bar{\pi}(x)=\pi(x, \infty)$ denotes the tail [similar notation is used for $\bar{F}(x)$, etc.], $S_{n}=X_{1}+\cdots+X_{n}, M=\max _{n=0,1, \ldots} S_{n}$ and $\tau(x)=$ $\inf \left\{n: S_{n}>x\right\}$; in (1.2), we used the standard fact that $W_{\infty}=\mathscr{D} M$. The analysis 
illustrates once more the role of big jumps: a large cycle maximum occurs as consequence of one big jump, whereas in the light-tailed case, it is preceded by a build-up period where the random walk evolves according to an exponentially twisted distribution ([2]).

Similar results hold for reflected Lévy processes in continuous time; we comment briefly upon this in Section 2.4.

1.2. Storage processes and ruin probabilities. The storage process $\left\{V_{t}\right\}_{t \geq 0}$ has i.i.d. upwards jump with common (heavy-tailed) distribution $B$ at Poisson times, say, with intensity $\beta$. Level 0 acts as reflecting barrier and in between jumps, the process moves downwards according to the (deterministic) ODE $\dot{x}(t)=-r(x(t))$, where $r(x)$ is the release rate at level $x$. A sufficient (and often close to necessary) condition for the existence of a limiting stationary distribution is that, for some $a<\infty$ and $\varepsilon>0$,

$$
r(x)>\beta \mu_{B}+\varepsilon, \quad x \geq a,
$$

where $\mu_{B}$ is the mean of $B$, and this will be assumed throughout. For more background see [20], [11] or [3], Chapter XIII.

The extremal behavior will be seen to be similar to that for the reflected random walk: the maximum of $\left\{V_{t}\right\}$ up to time $T$ behaves like the maximal jump in $[0, T]$. The extremal index $\theta$ depends on the asymptotic form of $r(x)$ and $\bar{B}(x)$; in some cases, we get $\theta=\infty$. The proofs of these results are again based upon the asymptotics of the cycle maximum. The difference from the random walk case is that there the asymptotic form of the stationary distribution $\pi$ was known (and played a crucial role), but that this is only true for storage processes in some quite special cases. Instead, we use a random walk comparison and auxiliary results proved in Section 2 for random walks and go the other way round: using a level crossing argument, the behavior within a cycle will be used to get the tail asymptotics of $\pi$. For light tails, this is only known for the special case of $B$ being exponential (here, in fact, an exact expression for $\pi$ is available; for various partial asymptotic results for more general light-tailed $B$ available via (1.4) below, see [7] and [29, 30]). For regularly varying $B$ and $r(x)=a+b x$, our result on $\pi$ is equivalent to a recent one of Klüppelberg and Stadtmüller [25].

The risk process $\left\{R_{t}\right\}_{t \geq 0}$ has i.i.d. downwards jump (claims) with common distribution $B$ at Poisson times, say, with intensity $\beta$, and in between jumps, the process moves upwards according to $\dot{x}(t)=+r(x(t))$, where the interpretation of $r$ is now the premium rate; in particular, when $r(x)=a+b x$, one can interpret $a$ as the pure premium and $b$ as the interest rate. The case $a=0$ is not purely academic: it arises in the "absolute ruin" problem considered by Dassios and Embrechts [12], and hence we will treat that as well, though it requires some extra effort. The infinite-horizon ruin probability is $\psi(x)=\mathbb{P}(\rho(x)<\infty)$, where $\rho(x)=\inf \{t: R(t)<0 \mid R(0)=x\}$ is the ruin time. It is known ([21], [8]) that the risk process and the storage process are 
connected by the formulas

$$
\begin{aligned}
\psi(x) & =\mathbb{P}\left(V_{\infty}>x\right), \\
\psi(x, T) & =\mathbb{P}(\rho(x) \leq T)=\mathbb{P}\left(V_{T}>x \mid V_{0}=0\right) .
\end{aligned}
$$

Thereby our results on storage processes apply to $\psi(u)$ as well [in fact, the result of [25] is formulated in terms of $\psi(u)$ ]. In addition, we will also show for the case $r(x)=a+b x$ that the conditional distribution of $\rho(x)$ given $\rho(x)<\infty$ has an exponential limit when properly normalized. Combining with the approximation for $\psi(x)$ provided by (1.4) and what was shown for storage processes, this yields an approximation for the finite-time horizon probability $\psi(x, T)$ (which in this setting is not even known for $B$ exponential; considering also that finite-time ruin probabilities are much harder to study than infinite-time ones in the classical case $b=0$, the proof to be given is, however, notably short).

1.3. Preliminaries. For the reflected random walk, we will assume throughout that $\bar{F}(x) \approx \bar{B}(x), x \rightarrow \infty$, for some distribution $B$ on $(0, \infty)$ which is subexponential, that is, satisfies $\overline{B^{* 2}}(x) / \bar{B}(x) \rightarrow 2, x \rightarrow \infty$; see, for example, [14] or [15] [the reason for introducing $B$ is the traditional setup of the subexponential area where one most often only works with distributions concentrated on $(0, \infty)$ ]. For example, this covers all $F$ with a tail which is regular varying, $\bar{F}(x)=L(x) / x^{\alpha}$ with $\alpha>1$ and $L(x)$ slowly varying, or Weibull- or lognormal-like. Let $F^{(x)}(y)=(F(x+y)-F(x)) / \bar{F}(x)$ be the overshoot distribution. A basic fact is that subexponentiality implies

$$
\lim _{x \rightarrow \infty} F^{(x)}(y)=0 \quad \text { uniformly in } y \in\left[0, y_{0}\right]
$$

for any $y_{0}<\infty$, that is, the overshoot $X-x \mid X>x$ converges in distribution to $\infty$.

In part, we will assume also that $F \in M D A(H)$ (maximum domain of attraction) with $H$ either Frechet or Gumbel. This yields a more precise asymptotic form of the overshoot distribution ([9], [17], [19]),

$$
\lim _{x \rightarrow \infty} \bar{F}^{(x)}(\gamma(x) y)=\lim _{x \rightarrow \infty} \frac{\bar{F}(x+\gamma(x) y)}{\bar{F}(x)}=\mathbb{P}\left(P_{\alpha}>y\right),
$$

where $\gamma(x)=\mathbb{E}[X-x \mid X>x]$ and $P_{\alpha}$ has a generalised Pareto distribution,

$$
\mathbb{P}\left(P_{\alpha}>x\right)=\left\{\begin{array}{lll}
(1+x /(\alpha-1))^{-\alpha}, & \alpha<\infty, & \\
e^{-x}, & \alpha=\infty, &
\end{array}\right.
$$

Here $\alpha<\infty$ corresponds to the Frechet case and $\alpha=\infty$ to the Gumbel case. Note that a different scale is used in much of the literature when $\alpha<\infty$; the present scale ensures that the mean is 1 . 
For the storage/risk process, the basic assumption is that the jump size distribution $B$ is subexponential. The relevance of heavy-tailed assumptions in risk theory is sometimes argued quite strongly; see, for example, [15].

\section{Reflected random walks.}

2.1. The cycle maximum. The process $\left\{W_{n}\right\}$ is regenerative with regenerative cycle

$$
\tau=\inf \left\{n>0: W_{n}=0\right\}=\inf \left\{n>0: S_{n} \leq 0\right\}
$$

(the descending ladder epoch). Write $\mu=\mathbb{E} \tau$ and $M_{\tau}=\max \left\{S_{n}: n=0, \ldots\right.$, $\tau-1\}$.

Theorem 2.1. Assume that (1.5) holds. Then

$$
\mathbb{P}\left(M_{\tau}>x\right)=\mathbb{P}(\tau(x)<\tau) \approx \mu \bar{F}(x), \quad x \rightarrow \infty .
$$

It follows readily from (1.2) and (1.5) that $\bar{\pi}(x) / \bar{F}(x) \rightarrow \infty$. Thus, Theorem 2.1 and (1.2) show that (in contrast to the light-tailed case) the tail of $M_{\tau}$ is lighter than the tail of $M=\max _{n=0,1, \ldots} S_{n}$ or, equivalently, the stationary r.v. $W_{\infty}$ with distribution $\pi$. Using the expression

$$
\theta=\lim _{x \rightarrow \infty} \frac{\mathbb{P}\left(M_{\tau}>x\right)}{\mu \bar{\pi}(x)}
$$

([28], page 380) for the extremal index, we get the following.

Corollary 2.1. The extremal index of $\left\{W_{n}\right\}$ is $\theta=0$.

For the proof of Theorem 2.1, we first introduce some notation. Define

$$
\begin{aligned}
& N_{1}\left(x, x_{0}\right)=\#\left\{n<\tau: S_{n} \leq x_{0}, S_{n+1}>x\right\}, \\
& p_{1}\left(x, x_{0}\right)=\mathbb{P}\left(S_{n+1}>x \text { for some } n<\tau \text { with } S_{n} \leq x_{0}\right), \\
& p_{2}\left(x, x_{0}\right)=\mathbb{P}\left(\tau(x)<\tau, x_{0} \leq S_{\tau(x)-1} \leq x\right) .
\end{aligned}
$$

[Note that the definitions of $p_{1}\left(x, x_{0}\right)$ and $p_{2}\left(x, x_{0}\right)$ are not symmetric in the sets $\left[0, x_{0}\right]$ and $\left(x_{0}, \infty\right)$.] Then,

$$
p_{1}\left(x, x_{0}\right) \leq \mathbb{P}\left(M_{\tau}>x\right) \leq p_{1}\left(x, x_{0}\right)+p_{2}\left(x, x_{0}\right) .
$$

Let further $R$ denote the occupation (renewal) measure of the random walk, $R(A)=\sum_{0}^{\infty} \mathbb{P}\left(S_{n} \in A\right)$. It is well known that $R[y, y+x] \leq a_{1}+a_{2} x$ for all $x, y$. Write further $m_{+}=\mathbb{E} X_{+}, m_{-}=\mathbb{E} X_{-}$(thus, $m=-\mathbb{E} X=m_{-}-m_{+}$). 
LEMMA 2.1. $\mathbb{E} N_{1}\left(x, x_{0}\right) \approx \mu \pi\left(x_{0}\right) \bar{F}(x)$.

Proof. Define $C(A)=\mathbb{E} \sum_{n=0}^{\tau-1} I\left(S_{n} \in A\right)$. By regenerative process theory, $C(A)=\mu \pi(A)$ and we get

$$
\begin{aligned}
N_{1}\left(x, x_{0}\right) & =\mathbb{E} \sum_{n=0}^{\tau-1} I\left(S_{n} \leq x_{0}, S_{n+1}>x\right) \\
& =\mathbb{E} \sum_{n=0}^{\tau-1} I\left(S_{n} \leq x_{0}\right) \bar{F}\left(x-S_{n}\right) \\
& =\int_{0}^{x_{0}} \bar{F}(x-y) C(d y)=\mu \int_{0}^{x_{0}} \bar{F}(x-y) \pi(d y) .
\end{aligned}
$$

Now just divide by $\bar{F}(x)$ and use dominated convergence justified by (1.5).

LEMMA 2.2. $p_{1}\left(x, x_{0}\right) \approx \mu \pi\left(x_{0}\right) \bar{F}(x)$.

Proof. After $\tau(x)$, the expected time $\left\{S_{n}\right\}$ spends in $\left(0, x_{0}\right)$ before hitting $(-\infty, 0]$ is bounded by $a_{1}+a_{2} x_{0}$. Hence, with $\alpha\left(x, x_{0}\right)=\left(a_{1}+a_{2} x_{0}\right) \bar{F}(x-$ $\left.x_{0}\right)$, we have

$$
\begin{aligned}
\mathbb{P}\left(N_{1}\left(x, x_{0}\right) \geq k+1 \mid N_{1}\left(x, x_{0}\right) \geq k\right) & \leq \alpha\left(x, x_{0}\right) \\
\mathbb{P}\left(N_{1}\left(x, x_{0}\right) \geq k+1\right) & \leq p_{1}\left(x, x_{0}\right) \alpha\left(x, x_{0}\right)^{k} \\
\mathbb{E}\left[N_{1}\left(x, x_{0}\right) ; N_{1}\left(x, x_{0}\right) \geq 2\right] & \leq p_{1}\left(x, x_{0}\right) \frac{\alpha\left(x, x_{0}\right)}{1-\alpha\left(x, x_{0}\right)}, \\
p_{1}\left(x, x_{0}\right) \leq \mathbb{E} N_{1}\left(x, x_{0}\right) & \leq p_{1}\left(x, x_{0}\right)+p_{1}\left(x, x_{0}\right) \frac{\alpha\left(x, x_{0}\right)}{1-\alpha\left(x, x_{0}\right)} .
\end{aligned}
$$

Now just note that $\alpha\left(x, x_{0}\right) \rightarrow 0$.

Letting first $x \rightarrow \infty$ and next $x_{0} \rightarrow \infty$ in (2.2), the following estimate will complete the proof of Theorem 2.1.

LeMma 2.3. $\lim _{x_{0} \rightarrow \infty} \lim \sup _{x \rightarrow \infty} p_{2}\left(x, x_{0}\right) / \bar{F}(x)=0$.

The proof is based upon a downcrossing argument. Define

$$
\begin{aligned}
D_{\tau}(x) & =\mathbb{E} \sum_{n=0}^{\tau-1} I\left(S_{n}>x, S_{n+1} \leq x\right), \\
D(x) & =\mathbb{E} \sum_{n=0}^{\infty} I\left(S_{n}>-x, S_{n+1} \leq-x\right) .
\end{aligned}
$$


LEMMA 2.4. $\lim _{x \rightarrow \infty} D(x)=m_{-} / m$.

Proof. Assume first that $F$ is nonlattice. It is well known that $R(d z-x)$ converges vaguely to Lebesgue measure normalized by $m$. Combining with the upper bounds for $R$ given above, similar estimates as in the proof of the key renewal theorem yield

$$
\begin{aligned}
D(x) & =\int_{-x}^{\infty} R(d y) F(-x-y)=\int_{0}^{\infty} R(d z-x) F(-z) \\
& \rightarrow \frac{1}{m} \int_{0}^{\infty} F(-z) d z=\frac{m_{-}}{m} .
\end{aligned}
$$

The lattice case is similar, though easier.

Proof of Lemma 2.3. By regenerative process theory,

$$
\begin{aligned}
\frac{D_{\tau}(x)}{\mu} & =\lim _{n \rightarrow \infty} \mathbb{P}\left(W_{n}>x, W_{n+1} \leq x\right) \\
& =\int_{x}^{\infty} \pi(d y) F(x-y) \\
& \approx \frac{1}{m} \int_{x}^{\infty} \bar{F}(y) d y \int_{-\infty}^{x-y} F(d z) \\
& =\frac{\bar{F}(x)}{m} \int_{-\infty}^{0} F(d z) \int_{x}^{x-z} \frac{\bar{F}(y)}{\bar{F}(x)} d y \\
& \approx \frac{\bar{F}(x)}{m} \int_{-\infty}^{0}|z| F(d z)=\bar{F}(x) \frac{m_{-}}{m},
\end{aligned}
$$

using dominated convergence and (1.5) in the fifth step.

On the other hand, the overshoot over $x$ after an upcrossing from a level less than or equal to $x_{0}$ converges in distribution to $\infty$ by (1.5), so that the expected subsequent number of downcrossings of level $x$ before $\left[0, x_{0}\right]$ is hit is approximately $m_{-} / m$ by Lemma 2.4 . Hence, we get asymptotically that

$$
\begin{aligned}
\mu \bar{F}(x) \frac{m_{-}}{m} & \approx D_{\tau}(x) \geq \mathbb{E} N_{1}\left(x, x_{0}\right) \frac{m_{-}}{m}+p_{2}\left(x, x_{0}\right) \\
& \approx \mu \bar{F}(x) \pi\left(x_{0}\right) \frac{m_{-}}{m}+p_{2}\left(x, x_{0}\right), \\
\limsup _{x \rightarrow \infty} \frac{p_{2}\left(x, x_{0}\right)}{\bar{F}(x)} & \leq \mu \bar{\pi}\left(x_{0}\right) \frac{m_{-}}{m} .
\end{aligned}
$$

For later use, we recollect some further auxiliary results on the behavior within a cycle. Let $\|\cdot\|$ denote the t.v. (total variation) distance between 
probability measures and define

$$
\begin{aligned}
F_{x}(A) & =\mathbb{P}(X \in A \mid X>x) \\
& =F^{(x)}(A-x)=\frac{\int_{A \cap(x, \infty)} F(d y)}{\bar{F}(x)} .
\end{aligned}
$$

Proposition 2.1. Let $K^{(x)}$ be the conditional distribution of $\left(S_{\tau(x)-1}\right.$, $\left.M_{\tau}-S_{\tau(x)}, X_{\tau(x)}\right)$ given $\tau(x)<\tau$. Then $\left\|K^{(x)}-\pi \times \pi \times F_{x}\right\| \rightarrow 0$. that

Proof. Let $x_{0}$ be fixed. It is easy to see from (1.5) and the definition of $F_{x}$ Define $\pi^{(x)}(A)=\mathbb{E} \sum_{0}^{\tau-1} I\left(W_{n} \in A, \tau(x)>n\right) / \mu$ and $\pi_{z}(A)=\mathbb{P}\left(\max _{n<\tau_{z}} S_{n} \in\right.$ $A$ ), where $\tau_{z}=\inf \left\{n: S_{n} \leq-z\right\}$. Then

$$
\left\|\pi-\pi^{(x)}\right\| \rightarrow 0, \quad\left\|\pi_{x}-\pi\right\| \leq \mathbb{P}(\tau(x)<\infty) \rightarrow 0 .
$$

Indeed, the second estimate follows by noting that $M$ and $\max _{n<\tau_{z}} S_{n} \in A$ only differ if $\left\{S_{n}\right\}$ upcrosses level 0 after downcrossing level $-x$. For the first, note that

$$
\begin{aligned}
\mu\left(\pi(A)-\pi^{(x)}(A)\right) & =\mathbb{E} \sum_{\tau(x)}^{\tau-1} I\left(W_{n} \in A\right) \\
& \leq \mathbb{E}[\tau-\tau(x) ; \tau(x)<\tau] \leq \mathbb{E}[\tau ; \tau(x)<\tau],
\end{aligned}
$$

which goes to 0 (uniformly in $A$ ) by monotone convergence.

Now let $A \subseteq[0, \infty)^{3}$ so that we can write

$$
\begin{aligned}
K^{(x)}(A)=\frac{1}{\mathbb{P}\left(M_{\tau}>x\right)} \int_{0}^{\infty} \mu \pi^{(x)}\left(d y_{1}\right) & \int_{x-y_{1}}^{\infty} F\left(d y_{3}\right) \\
& \times \int_{0}^{\infty} \pi_{y_{1}+y_{3}}\left(d y_{2}\right) I\left(\left(y_{1}, y_{2}, y_{3}\right) \in A\right) .
\end{aligned}
$$

Define

$$
\begin{aligned}
& A_{1}=A \cap\left(\left[0, x_{0}\right] \times[0, \infty)^{2}\right), \\
& A_{2}=A \cap\left(\left(x_{0}, \infty\right) \times[0, \infty)^{2}\right) .
\end{aligned}
$$

Using (2.3) and (2.4), it is easy to see that $K^{(x)}\left(A_{1}\right)-\pi \times \pi \times F_{x}\left(A_{1}\right) \rightarrow 0$ uniformly in $A_{1}$. Hence,

$$
\begin{aligned}
\limsup _{x \rightarrow \infty}\left\|K^{(x)}-\pi \times \pi \times F_{x}\right\| & =\limsup _{x \rightarrow \infty} \sup _{A}\left|K^{(x)}\left(A_{2}\right)-\pi \times \pi \times F_{x}\left(A_{2}\right)\right| \\
& \leq \limsup _{x \rightarrow \infty} \sup _{A}\left(K^{(x)}\left(A_{2}\right)+\pi \times \pi \times F_{x}\left(A_{2}\right)\right) \\
& \leq \limsup _{x \rightarrow \infty}\left(\frac{p_{2}\left(x, x_{0}\right)}{\mu \bar{F}(x)}+\bar{\pi}\left(x_{0}\right)\right) \\
& \leq \bar{\pi}\left(x_{0}\right)\left(\frac{m_{-}}{m}+1\right) .
\end{aligned}
$$

Let $x_{0} \rightarrow \infty$. 
Corollary 2.2. $\left\|\mathbb{P}\left(M_{\tau} \in \cdot\right)-\pi * \pi * F_{x}\right\| \rightarrow 0$.

[The result is not used in the sequel but is of some independent interest as a sharpening of Theorem 2.1.]

Lemma 2.5. $\mathbb{P}\left(\max _{l<\tau, l \neq \tau(x)} X_{l}>X_{\tau(x)} \mid M_{\tau}>x\right) \rightarrow 0$.

Proof. By Proposition 2.1, the conditional probability that $X_{\tau(x)}>x$ goes to 1 . Hence,

$$
\mathbb{P}\left(\max _{l<\tau(x)} X_{l}>X_{\tau(x)} \mid M_{\tau}>x\right) \rightarrow 0
$$

Furthermore,

$$
\mathbb{P}\left(\max _{\tau(x)<l<\tau} X_{l}>x\right) \leq\left(a_{1}+a_{2} x\right) \bar{F}(x) \rightarrow 0,
$$

since $m_{+}<\infty$.

2.2. Extremal behavior. Define $F^{(n)}(x)=\mathbb{P}\left(M_{n} \leq x\right)$, where $M_{n}=$ $\max _{k=0, \ldots, n} W_{n}, G(x)=\mathbb{P}\left(M_{\tau} \leq x\right)$, and let $\|\cdot\|$ denote the supremum norm between distributions. The following lemma is valid for any regenerative process $\left\{W_{n}\right\}$ (only part (i) is needed at present). We recall the following basic estimate ([26], page 13): if $F$ is any distribution and $\tau \in[0, \infty]$, then

$$
F^{n}\left(u_{n}\right) \rightarrow e^{-\tau} \Leftrightarrow n \bar{F}\left(u_{n}\right) \rightarrow \tau .
$$

LEMMA 2.6. (i) $\left\|F^{(n)}-G^{n / \mu}\right\| \rightarrow 0$.

(ii) Assume that $\left\{W_{n}\right\}$ is regenerative wrt a different renewal process, say, with generic cycle $\tau_{*}$ with mean $\mu_{*}$. Then $\bar{G}_{*}(x) \approx\left(\mu_{*} / \mu\right) \bar{G}(x)$.

(iii) Let $A_{n} \downarrow \varnothing$ be a sequence of events depending only on $\left\{W_{n}\right\}_{n<\tau}, A_{n}(k)$ the event corresponding to $A_{n}$ but defined in terms of the kth cycle rather than the first and $p_{n}=\mathbb{P}\left(A_{n}\right)$. Then if $\underline{\sigma}_{n}, \bar{\sigma}_{n}$ are the times at which the first cycle $k$ in which $A_{n}$ occurs starts, resp. ends, it holds that both $p_{n} \underline{\sigma}_{n} / \mu$ and $p_{n} \bar{\sigma}_{n} / \mu$ have a limiting standard exponential distribution as $n \rightarrow \infty$.

Proof. Part (i) is given on page 375 of [28]. Part (ii) is an easy consequence of (i). Indeed, assume that $\mu_{*} \bar{G}\left(u_{k}\right) / \mu \bar{G}_{*}\left(u_{k}\right) \rightarrow \delta \neq 1$ for some sequence $\left\{u_{k}\right\}$ with $u_{k} \rightarrow \infty$. Then we can choose first $\tau \in(0, \infty)$ arbitrarily and next, $\left\{n_{k}\right\}$ such that

$$
\frac{n_{k}}{\mu_{*}} \bar{G}_{*}\left(u_{k}\right) \rightarrow \tau, \quad \frac{n_{k}}{\mu} \bar{G}\left(u_{k}\right) \rightarrow \delta \tau .
$$

By (i) and (2.5), this implies that $\mathbb{P}\left(M_{n_{k}} \leq u_{k}\right)$ has limits both $e^{-\tau}$ and $e^{-\delta \tau}$, a contradiction.

Part (iii) is standard (e.g., [18] or [23]).

Now return to the reflected random walk. 
Theorem 2.2. Let $0 \leq \kappa \leq \infty$. Then, for any sequence $u_{n} \uparrow \infty, F^{(n)}\left(u_{n}\right) \rightarrow$ $e^{-\kappa}$ if and only if $F^{n}\left(u_{n}\right) \rightarrow e^{-\kappa}$.

Proof. Using Lemma 2.6(i) for (a), (2.5) for (b) and (d) and Theorem 2.1 for (c), we get

$$
\begin{aligned}
F^{(n)}\left(u_{n}\right) \rightarrow e^{-\kappa} & \stackrel{(\mathrm{a})}{\Leftrightarrow} G^{n / \mu}\left(u_{n}\right) \rightarrow e^{-\kappa} \stackrel{(\mathrm{b})}{\Leftrightarrow} \frac{n}{\mu} \bar{G}\left(u_{n}\right) \rightarrow \kappa \\
& \stackrel{(\mathrm{c})}{\Leftrightarrow} n \bar{F}\left(u_{n}\right) \rightarrow \kappa \stackrel{(\mathrm{d})}{\Leftrightarrow} F^{n}\left(u_{n}\right) \rightarrow e^{-\kappa} .
\end{aligned}
$$

COROLlaRY 2.3. For any sequences $\left\{a_{n}\right\},\left\{b_{n}\right\}$ of constants, $F^{(n)}\left(x / a_{n}+b_{n}\right)$ $\rightarrow H(x)$ for all $x$ if and only if $F^{n}\left(x / a_{n}+b_{n}\right) \rightarrow H(x)$ for all $x$.

Theorem 2.3. Assume that $F$ is continuous. Then

$$
M_{n}-\max _{k=0, \ldots, n} X_{k} \rightarrow W_{\infty}^{(1)}+W_{\infty}^{(2)}
$$

in total variation where $W_{\infty}^{(1)}, W_{\infty}^{(2)}$ are independent with common distribution $\pi$.

Proof. Define $\tilde{X}_{i}$ as the maximal jump in the $i$ th cycle and write the cycle maximum as $M_{\tau_{i}}=\tilde{X}_{i}+V_{i}$. Then, by Proposition 2.1 and Lemma 2.5, the conditional distribution of $V_{i}$ given $M_{\tau_{i}}>x$ converges in t.v. to that of $W_{\infty}^{(1)}+W_{\infty}^{(2)}$.

For $K<\infty$, choose a sequence $\left\{u_{n}^{(K)}\right\}$ such that $n(1+\varepsilon) \bar{F}\left(u_{n}^{(K)}\right) \rightarrow K$, let $I(n, K)$ be the set of indices $i \leq n(1+\varepsilon) / \mu$ of cycles with $M_{\tau_{i}}>u_{n}^{(K)}$ and $k=k(n, K)$ the index of the cycle with the maximal $M_{\tau_{i}}$. Then, by Theorem 2.1 and Lemma 2.6(iii), the number $|I(K, n)|$ of elements of $I(K, n)$ is asymptotically Poisson $(K)$, and thus by choosing $\varepsilon$ small enough, $K, L$ large enough, we can obtain that, for $n$ large enough, we have $\mathbb{P}\left(F_{1}\right)>1-\delta$, where

$$
F_{1}=\left\{2 \leq|I(K, n)| \leq L, M_{\tau_{i}}=M_{n}\right\} .
$$

Next we note that $\mathbb{P}\left(\tilde{X}_{k}>u_{n}^{(K)}\right) \rightarrow 1$ by Proposition 2.1 . Thus cycles with $i \notin I(n, K)$ cannot contribute to $\max _{k<n} X_{k}$. For the ones with $i \in I(n, K)$, Lemma 2.5 shows that the only contribution comes from $\tilde{X}_{i}$. It follows that we have $\mathbb{P}\left(F_{2}\right)>1-2 \delta$ for $n$ large enough, where

$$
F_{2}=F_{1} \cap\left\{\max _{i \in I(K, n)} \tilde{X}_{i}=\max _{k \leq n} X_{k}\right\}, \quad F_{3}=F_{2} \cap\left\{\max _{i \in I(K, n)} \tilde{X}_{i}=\tilde{X}_{k}\right\} .
$$

To show that we have also $\mathbb{P}\left(F_{3}\right)>1-3 \delta$ for $n$ large enough, it suffices to show that, if $M_{\tau_{1}}>u_{n}, \ldots, M_{\tau_{L}}>u_{n}$ and $M_{\tau_{L}}=\max _{i=1, \ldots, L} M_{\tau_{i}}$, then $\mathbb{P}\left(\tilde{X}_{L}=\max _{i=1, \ldots, L} \tilde{X}_{i}\right) \rightarrow 1$. Define $\tilde{M}=\max _{i=1, \ldots, L-1} M_{\tau_{i}}$. Then the conditional distribution of $M_{\tau_{L}}$ given $M_{\tau_{1}}, \ldots, M_{\tau_{L-1}}$ is $\mathbb{P}\left(M_{\tau} \stackrel{\tau_{i}}{\in} \cdot \mid M_{\tau}>\tilde{M}\right)$, and since $\tilde{M}>u_{n} \rightarrow \infty$, it follows by Theorem $2.1_{\tilde{X}_{L-1}}$ and (1.5) that $M_{\tau_{L}}-\tilde{M} \rightarrow \infty$. Since $V_{L}$ has a finite limit, we have also $\tilde{X}_{L}-\tilde{M} \rightarrow \infty$ and hence $\tilde{X}_{L}-$ $\max _{i=1, \ldots, L-1} \tilde{X}_{i} \rightarrow \infty$. 
Now just note that on $F_{3}, M_{n}-\max _{l=0, \ldots, n} X_{l}=V_{k}$, and it was noted above that $V_{k}$ has the desired asymptotic distribution.

2.3. Exceedances. The random set $\left\{k=0, \ldots, n: W_{k}>u_{n}\right\}$ is one of the classical objects of study of extreme value theory. The traditional situation with extremal index $\theta>0$ is that $\left\{k=0, \ldots, n: W_{k}>u_{n}\right\}$ is contracted to $[0,1]$ and that one gets a compound Poisson limit with the compounding distribution depending on the process in question (see [26], Chapter 5; [28], pages 377-378). Here we will need an additional scaling involving $\gamma(x)=$ $\mathbb{E}[X-x \mid X>x]$ and obtain a compound Poisson limit with the compounding distribution only depending on the maximum domain of attraction. To this end, define a point process $N_{n}$ on $[0,1]$ by

$$
N_{n}(A)=\#\left\{k=0, \ldots, n: k / n \in A, W_{k}>u_{n}\right\} .
$$

THEOREM 2.4. Assume that $F \in M D A(H)$ with normalizing constants $a_{n}, b_{n}$ and $u_{n}=x / a_{n}+b_{n}$. Then $m N_{n} / \gamma\left(u_{n}\right)$ converges in distribution to a compound Poisson process with intensity - $\log H(x)$ and compounding distribution $\mathbb{P}\left(P_{\alpha} \leq x\right)$, where $\alpha<\infty$ corresponds to the Frechet case and $\alpha=\infty$ to the Gumbel case.

Proof. Define

$$
\begin{aligned}
T(x, y) & =\#\left\{n<\tau: W_{n}>x \mid S_{\tau(x)}=x+y\right\}, \\
T(x) & =\#\left\{n<\tau: W_{n}>x \mid M_{\tau}>x\right\} .
\end{aligned}
$$

Then $T(x, y) / y \rightarrow 1 / m$ as $y \rightarrow \infty$, and hence by Proposition 2.1 and (1.6), $m T(x) / \gamma(x) \rightarrow_{\mathscr{D}} P_{\alpha}$. The result follows by letting $x=u_{n}$ and invoking that cycles with $M_{\tau}>u_{n}$ occur asymptotically according to a Poisson process (cf. Lemma 2.6(iii) or [28]); the asymptotic intensity for $N_{n}$ is the expected number of events in $[0,1]$, that is,

$$
n \cdot \frac{\mathbb{P}\left(M_{\tau}>u_{n}\right)}{\mu} \approx n \bar{F}\left(u_{n}\right) \approx-\log H(x),
$$

as asserted [the last $\approx$ follows from $F^{n}\left(u_{n}\right) \rightarrow H(x)$ ].

2.4. Reflected Lévy processes in continuous time. Assume now that $\left\{S_{t}\right\}_{t \geq 0}$ is a Lévy process in continuous time, that is, $S_{t}=\alpha t+\sigma B_{t}+J_{t}$, where $\left\{B_{t}\right\}$ is standard Brownian motion and $\left\{J_{t}\right\}$ a pure jump process with Lévy measure $\varphi(d x)$. Assume further that the paths are right-continuous, that

$$
m=-\alpha-\int_{-\infty}^{\infty} x \varphi(d x)>0
$$

and that $\bar{\varphi}(x) \approx \bar{G}(x)$ for some subexponential $G$. This covers, for example, stable processes of index less than 2 , where $\bar{\varphi}(x)$ is regularly varying. Let $W_{t}=S_{t}-\inf _{0 \leq v \leq t} S_{v}$ be the reflected Lévy process and define the cycle as

(2.6) $\tau= \begin{cases}\inf \left\{t>0: S_{t}<\inf _{0 \leq v \leq t} S_{v}, \sup _{v \leq t} W_{v}>1\right\}, & \varphi(-\infty, 0)>0, \\ \inf \left\{t>0: S_{t} \leq \inf _{0 \leq v \leq t} S_{v}, \sup _{v \leq t} W_{v}>1\right\}, & \varphi(-\infty, 0)=0,\end{cases}$ 
(note that taking the cycle as $\inf \left\{t>0: W_{t}=0\right\}$ may lead to $\tau=0$; the distinction between the two cases in (2.6) is needed to ensure the estimate $h \mu_{h} \downarrow \mu$ below).

Corollary 2.4. $\mathbb{P}\left(M_{\tau}>x\right) \approx \mu \bar{\varphi}(x)$.

Proof. It follows exactly as in discrete time that

$$
\liminf _{x \rightarrow \infty} \frac{\mathbb{P}\left(M_{\tau}>x\right)}{\bar{\varphi}(x)} \geq \mu .
$$

For lim sup $\leq$, we use a discrete approximation via $W_{n}^{(h)}=S_{n h}-$ $\inf _{k=0, \ldots, n} S_{k h}$. Let $\tau_{h}=\inf \left\{k: k h>\tau, W_{k}^{(h)}=0\right\}$. Considering the two cases in (2.6) separately shows that $h \tau_{h} \downarrow \tau$ a.s. as $h \downarrow 0$ and hence $h \mu_{h} \downarrow \mu$.

Decomposing $\left\{S_{t}\right\}$ into an independent sum of Lévy processes with Lévy measures $\varphi(A \cap(-\infty, 1]))$, resp. $\varphi(A \cap(1, \infty))$, the first term has an exponentially bounded tail at time $h$ and the second one a tail which is asymptotically $h \bar{\varphi}(x)$, so that

$$
\mathbb{P}\left(S_{h}>x\right) \approx h \bar{\varphi}(x) .
$$

Noting that $W_{n+1}^{(h)}=\left(W_{n}^{(h)}+S_{(n+1) h}-S_{n h}\right)^{+}$, the proof in discrete time carries easily over to show $\mathbb{P}\left(M_{\tau_{h}}^{(h)}>x\right) \approx \mu_{h} h \bar{\varphi}(x)$ (the modification of the cycle plays no role in the argument). Let $\delta>0$. Then, for all sufficiently small $h$, $\mathbb{P}\left(S_{t}>-1\right) \geq 1-\delta$ for all $t \leq h$, so that

$$
\mathbb{P}\left(M_{\tau_{h}}^{(h)}>x\right) \approx \mathbb{P}\left(M_{\tau_{h}}^{(h)}>x-1\right) \geq \mathbb{P}\left(M_{\tau}>x\right)(1-\delta) .
$$

Given Corollary 2.4, the analysis and results of the rest of Sections 2.1-2.3 carry easily over to continuous time. The form of the results is obvious and we omit a formal statement.

Representing the stationary distributions as

$$
W_{\infty}=\max _{t \geq 0} S_{t}, \quad W_{\infty}^{(h)}=\max _{k=0,1, \ldots} S_{k h},
$$

we have $W_{\infty} \geq W_{\infty}^{(h)}$, so that (1.2) and (2.7) yield

$$
\mathbb{P}\left(W_{\infty}>x\right) \geq \mathbb{P}\left(W_{\infty}^{(h)}>x\right) \approx \frac{1}{-\mathbb{E} S_{h}} \int_{x}^{\infty} h \bar{\varphi}(y) d y=\frac{1}{m} \int_{x}^{\infty} \bar{\varphi}(y) d y .
$$

The converse inequality follows as in the proof of Corollary 2.4 , and hence we obtain the following result for which we have no reference (note that the argument of [16] crucially relies on the ladder height renewal process, which may not make sense in continuous time for reasons similar to why we could not take $\left.\tau=\inf \left\{t: W_{t}=0\right\}\right)$.

Corollary 2.5. $\mathbb{P}\left(W_{\infty}>x\right) \approx(1 / m) \int_{x}^{\infty} \bar{\varphi}(y) d y$.

It may also be noted that the analysis of Section 2.1 yields certain refinements and sharpenings of the study of Asmussen and Klüppelberg [6] of the excursions of the $\mathrm{M} / \mathrm{G} / 1$ workload process, corresponding to $\alpha=-1$, 
$\sigma^{2}=0$ and $\varphi=\beta B$, where $\beta$ is the arrival intensity and $B$ the service time distribution. More precisely, the analysis of the upcrossings of level $x$ within a cycle, expressed through the study of $p_{1}\left(x, x_{0}\right)$ and $p_{2}\left(x, x_{0}\right)$, shows that:

1. The assumption of [6] that $B$ belongs to the class $\mathscr{S}^{*}$ introduced in [24] is not necessary; it suffices that $B$ is subexponential.

2. In the classification of excursions over a large level $x$ into two types, the ones starting with a jump from a level which is $O(1)$ and the ones where this level is close to $x$, one can view the last type of excursions as aftereffects of one of the first type: within a cycle, the first upcrossing of $x$ occurs asymptotically from a $O(1)$ level, and the remaining upcrossings after the subsequent downcrossing from a level which is $x-O(1)$. By regenerative process theory, this description of the cycle carries over to the stationary setting considered in [6].

3. Storage processes. Consider the storage process $\left\{V_{t}\right\}$ and define

$$
t(x, z)=\int_{x}^{x+z} \frac{1}{r(y)} d y
$$

as the time needed for the solution of $\dot{x}(t)=-r(x(t))$ to reach level $x$ starting from $x+z$.

3.1. The cycle maximum and extremal behavior. If $t(0, z)<\infty$ for all $z>0$, the natural choice of the regenerative cycle is the first return to 0 after an excursion in $(0, \infty)$ (thus, it starts with an exponential period where $\left.V_{t}=0\right)$

$$
\tau=\inf \left\{t>0: \sup _{s \leq t} V_{s}>0, V_{t}=0 \mid V_{0}=0\right\} .
$$

Otherwise, we choose $\varepsilon>0$ such that $t(\varepsilon, z)<\infty$ for all $z>0$, start the storage process with $V_{0}=\varepsilon$ and let $\tau$ be the time of the first downcrossing of level $\varepsilon$,

$$
\tau=\inf \left\{t>0: \sup _{s \leq t} V_{s}>\varepsilon, V_{t}=\varepsilon \mid V_{0}=\varepsilon\right\} .
$$

Note, however, that by Lemma 2.6(ii), the particular choice of the regenerative cycle is unimportant for the study of the tail of the cycle maximum $M_{\tau}=\max _{t<\tau} V_{t}$. Write again $\mu=\mathbb{E} \tau, \tau(x)=\inf \left\{t: V_{t}>x\right\}$.

\section{THEOREM 3.1.}

$$
\mathbb{P}\left(M_{\tau}>x\right)=\mathbb{P}(\tau(x)<\tau) \approx \mu \beta \bar{B}(x), \quad x \rightarrow \infty .
$$

Given Theorem 3.1, the following result on extremal behavior of $\left\{V_{t}\right\}$ is obtained by minor variants of arguments of Sections 2.2-2.3. Let $U_{1}, U_{2}, \ldots$ be the jumps (i.i.d. governed by $B$ ). 
Corollary 3.1. For any sequence $u_{n} \uparrow \infty, \mathbb{P}\left(\max _{t \leq T} V_{t} \leq u_{n}\right) \rightarrow e^{-\kappa}$ if and only if $\mathbb{P}\left(\max _{k=1, \ldots,\lfloor\beta T]} U_{k} \leq u_{n}\right) \rightarrow e^{-\kappa}$.

Proof. Let $M_{T}$ be the number of jumps (Poisson events) before $T$. Applying Lemma 2.6(i) to the regenerative process formed by the next jump, it is easily seen that we can approximate $\max _{k=1, \ldots, M_{T}} U_{k}$ by $\max _{k=1, \ldots,\lfloor\beta T\rfloor} U_{k}$. The rest of the proof is as for the reflected random walk case.

We return to further aspects of extreme value theory in Section 5.

Proof of Theorem 3.1. We first consider the case where $r(x) \geq b$ for all $x$ [not only for all sufficiently large $x$ as in (1.3)], where $b$ is a constant with $b>\beta \mu_{B}$. As in the proof of Theorem 2.1, define

$$
\begin{aligned}
& N_{1}\left(x, x_{0}\right)=\#\left\{t<\tau: V_{t-} \leq x_{0}, V_{t}>x\right\}, \\
& p_{1}\left(x, x_{0}\right)=\mathbb{P}\left(V_{t}>x \text { for some } t<\tau \text { with } V_{t-} \leq x_{0}\right) .
\end{aligned}
$$

Since the expected passage time from $x$ to 0 is bounded by $x /\left(b-\beta \mu_{B}\right)$, it follows as in Lemma 2.2 that $p_{1}\left(x, x_{0}\right) \approx \mathbb{E} N_{1}\left(x, x_{0}\right)$. But by dominated convergence and (1.5),

$$
\mathbb{E} N_{1}\left(x, x_{0}\right)=\mu \int_{0}^{x_{0}} \beta \bar{B}(x-y) \pi(d y) \approx \mu \beta \bar{B}(x) \pi\left(x_{0}\right),
$$

and thus, $\liminf \mathbb{P}\left(M_{\tau}>x\right) / \mu \beta \bar{B}(x) \geq 1$.

For the converse inequality, we use a comparison with a process $\left\{V_{b, t}\right\}$ with the constant release rate $r_{b}(x)=b$ (we use obvious notation like $\mu_{b}$, etc.). This process is (up to a time change) the $\mathrm{M} / \mathrm{G} / 1$ virtual waiting time process, that is, a continuous-time reflected Lévy process. Since $V_{t} \leq V_{b, t}$ for all $t,\left\{V_{b, t}\right\}$ regenerates as well in state 0 at time $\tau$ : thus,

$$
\mathbb{P}\left(M_{\tau}>x\right) \leq \mathbb{P}\left(M_{\tau, b}>x\right) \approx \mu \beta \bar{B}(x),
$$

using Lemma 2.6(ii) and Corollary 2.4 in the last step.

Now consider the general case (1.3). Again, $\lim \inf \mathbb{P}\left(M_{\tau}>x\right) / \mu \beta \bar{B}(x) \geq 1$ is easy: the only modification from the case $r(x) \geq b$ which is needed, is to bound the expected passage time from $x$ to 0 by $(x-a) / \varepsilon+\mathbb{E}\left(\tau \mid V_{0}=a\right)$. The proof of lim sup $\leq$ utilizes the comparison $V_{t} \leq a+V_{t}^{*}$, where $a$ is as in (1.3) and $\left\{V_{t}^{*}\right\}$ is a storage process with $r^{*}(x)=r(x+a)$. Denote the cycle means by $\mu, \mu_{*}$ and the distributions of the cycle maximum by $G, G_{*}$. Let $T=T(x)$ vary with $x$ in such a way that $T(x) \bar{G}_{*}(x) \rightarrow \mu_{*} \kappa$ for some $\kappa \in(0, \infty)$; since it has been shown that $\bar{G}_{*}(x) \approx \mu_{*} \beta \bar{B}(x)$, we have $T(x) \approx$ $\kappa /(\beta \bar{B}(x))$. Then also $T(x) \bar{G}_{*}(x-a) \rightarrow \mu_{*} \kappa$ by (1.5), and by Lemma 2.6(i),

$$
\begin{aligned}
\mathbb{P}\left(\max _{t \leq T} V_{t}^{*} \leq x-a\right) & \rightarrow e^{-\kappa}, \quad \liminf _{x \rightarrow \infty} \mathbb{P}\left(\max _{t \leq T} V_{t} \leq x\right) \geq e^{-\kappa}, \\
\liminf _{x \rightarrow \infty} G^{T / \mu}(x) \geq e^{-\kappa}, & \limsup _{x \rightarrow \infty} T \bar{G}(x) \leq \mu \kappa .
\end{aligned}
$$


Inserting the asymptotic form of $T=T(x)$ shows that $\mu \beta \bar{B}(x)$ is an asymptotic upper bound for $\bar{G}(x)=\mathbb{P}\left(M_{\tau}>x\right)$.

3.2. The tail of the stationary distribution. Our second application of Theorem 3.1 is to obtain the tail behavior of the stationary distribution; more precisely, the asymptotics of the density $f(x)$.

TheOREM 3.2. (i) Assume that $r(x) \rightarrow r(\infty), x \rightarrow \infty$, where $\beta \mu_{B}<r(\infty)<\infty$. Then

$$
f(x) \approx \frac{\beta}{r(\infty)-\beta \mu_{B}} \bar{B}(x) .
$$

(ii) Assume that $r(x) \rightarrow \infty$. Then

$$
f(x) \approx \frac{\beta \bar{B}(x)}{r(x)} .
$$

Proof. Define $D(x)$ as the steady-state rate of downcrossings of level $x$ and $D_{\tau}(x)$ as the expected number of downcrossings of level $x$ during a cycle. Then $D(x)=D_{\tau}(x) / \mu, D(x)=f(x) r(x)$. Further, the conditional distribution of the number of downcrossings of $x$ during a cycle given $\tau(x)<\tau$ is geometric with parameter $p(x)=\mathbb{P}\left(\tau(x)<\tau \mid V_{0}=x\right)$. Hence,

$$
f(x) r(x)=D(x)=\frac{D_{\tau}(x)}{\mu}=\frac{\mathbb{P}\left(M_{\tau}>x\right)}{\mu(1-p(x))} \approx \frac{\beta \bar{B}(x)}{1-p(x)} .
$$

If $r=r(x)$ does not depend on $x$, it is standard that the extension $\left\{V_{t}^{*}\right\}$ of $\left\{V_{t}\right\}$ to $(-\infty, \infty)$ obtained by deleting the reflection at 0 has probability $\beta \mu_{B} / r$ of ever upcrossing 0 when $V_{0}^{*}=0$, and hence $p(x) \rightarrow \beta \mu_{B} / r$. The same conclusion is easily seen to hold if $r(x) \rightarrow r$. Letting $r=r(\infty)$ and inserting in (3.1), (i) follows. Case (ii) is similar though slightly easier [here $p(x) \rightarrow 0$ ].

4. Ruin probabilities. Consider the risk process $\left\{R_{t}\right\}$ and define the ruin time $\rho(x)$ as $\inf \left\{t: R_{t}<0 \mid R_{0}=x\right\}$. Combining (1.4) and Theorem 3.2 yields the following.

Corollary 4.1. (i) Assume that $r(x) \rightarrow r(\infty), x \rightarrow \infty$, where $\beta \mu_{B}<r(\infty)<$ $\infty$. Then

$$
\begin{aligned}
\psi(x) & =\mathbb{P}(\rho(x)<\infty) \\
& \approx \frac{\beta}{r(\infty)-\beta \mu_{B}} \int_{x}^{\infty} \bar{B}(y) d y, \quad x \rightarrow \infty .
\end{aligned}
$$

(ii) Assume that $r(x) \rightarrow \infty$. Then

$$
\psi(x)=\mathbb{P}(\rho(x)<\infty) \approx \int_{x}^{\infty} \frac{\beta \bar{B}(y)}{r(y)} d y .
$$


From now on, we concentrate on the case $r(x)=a+b x$ with $b>0$, so that by (ii), $\psi(x) \approx \bar{G}(x)$, where

$$
\bar{G}(x)=\beta \int_{x}^{\infty} \frac{1}{a+b y} \bar{B}(y) d y \approx \frac{\beta}{b} \int_{x}^{\infty} \frac{1}{y} \bar{B}(y) d y .
$$

We will impose a slightly stronger condition on $B$ than just being subexponential: in addition, we require that either $\bar{B}(x)$ is regularly varying [which is equivalent to $B \in M D A\left(\Phi_{\alpha}\right)$ ] or that $B \in M D A(\Lambda)$. In the regularly varying case, the constants $\gamma(x)$ in (1.6) satisfy $\gamma(x) \approx x /(\alpha-1)$, while if $B \in$ $\operatorname{MDA}(\Lambda)$, it is easy to see from results of [9] that $\gamma(x) / x \rightarrow 0$. We note the following analytical properties of $G$.

Proposition 4.1. (i) In the regularly varying case,

$$
\bar{G}(x) \approx \frac{\beta L(x)}{\alpha b x^{\alpha}}=\frac{\beta}{\alpha b} \bar{B}(x) ;
$$

(ii) if $B \in M D A(\Lambda)$, then

$$
\bar{G}(x) \approx \frac{\beta \gamma(x)}{b x} \bar{B}(x) ;
$$

(iii) $\bar{G}(x+\gamma(x) z) / \bar{G}(x) \rightarrow \mathbb{P}\left(P_{\alpha}>z\right)$.

PRoof. Part (i) is straightforward. For (ii), we first recall ([27], Proposition 1.17) that the integrated tail $\bar{B}_{0}(x)=\int_{x}^{\infty} \bar{B}(y) d y=\gamma(x) \bar{B}(x)$ again satisfies (1.6), with the same $\gamma(x)$. Using $\gamma(x) / x \rightarrow 0$ in the first step, we then get

$$
\begin{aligned}
\int_{x}^{x+\gamma(x) z} \frac{1}{y} \bar{B}(y) d y & \approx \frac{1}{x}\left(B_{0}(x+\gamma(x) z)-B_{0}(x)\right) \\
& \approx \frac{1}{x} B_{0}(x) \mathbb{P}\left(P_{\infty} \leq z\right)=\frac{\gamma(x)}{x} \bar{B}(x) \mathbb{P}\left(P_{\infty} \leq z\right), \\
\int_{x+\gamma(x) z}^{\infty} \frac{1}{y} \bar{B}(y) d y & \leq \frac{1}{x} \bar{B}_{0}(x+\gamma(x) z) \approx \frac{1}{x} \bar{B}_{0}(x) \mathbb{P}\left(P_{\infty}>z\right) \\
& \approx \frac{\gamma(x)}{x} \bar{B}(x) \mathbb{P}\left(P_{\infty}>z\right) .
\end{aligned}
$$

Let first $x \rightarrow \infty$ and next $z \rightarrow \infty$.

Part (iii) follows immediately from (i) in the regularly varying case. If $B \in M D A(\Lambda)$, use (ii) and

$$
\gamma(x+\gamma(x) z)=\frac{\bar{B}_{0}(x+\gamma(x) z)}{\bar{B}(x+\gamma(x) z)} \approx \frac{\bar{B}_{0}(x) \mathbb{P}\left(P_{\infty}>z\right)}{\bar{B}(x) \mathbb{P}\left(P_{\infty}>z\right)}=\gamma(x) .
$$

We next consider the distribution of the ruin time and will exhibit constants $\delta(x)$ such that the conditional distribution of $\delta(x) \rho(x)$ given $\rho(x)<\infty$ has an exponential limit. Let $d_{x}(t)$ be the solution of $\dot{d}(t)=r(d(t))$ satisfying $d_{x}(0)=x$. 
Lemma 4.1. Define

$$
\begin{aligned}
T & =T(x)=d_{x}^{-1}(x+\gamma(x) y) \\
& =\int_{x}^{x+\gamma(x) y} \frac{1}{a+b z} d z=\frac{1}{b} \log \left(1+\frac{\gamma(x) y}{x+a / b}\right) .
\end{aligned}
$$

Then $\psi(x, T)=\mathbb{P}(\rho(x) \leq T) \approx \psi(x) \mathbb{P}\left(P_{\alpha} \leq y\right)$.

Proof. In the absence of claims in $[0, t], R_{t}=d_{x}(t)$ when $R_{0}=x$, and hence always $R_{t} \leq d_{x}(t)$. This yields immediately that

$$
\begin{aligned}
\psi(x)-\psi(x, T) & =\mathbb{P}(T<\rho(x)<\infty)=\mathbb{E}\left[\psi\left(R_{T}\right) ; \rho(x)>T\right] \\
& \geq \psi\left(d_{x}(T)\right) \mathbb{P}(\rho(x)=\infty) \\
& =\psi(x+\gamma(x) y)(1-\psi(x)), \\
\limsup _{x \rightarrow \infty} \frac{\psi(x, T)}{\psi(x)} & \leq 1-\lim _{x \rightarrow \infty} \frac{\psi(x+\gamma(x) y)}{\psi(x)}=1-\lim _{x \rightarrow \infty} \frac{\bar{G}(x+\gamma(x) y)}{\bar{G}(x)} \\
& =1-\mathbb{P}\left(P_{\alpha}>y\right)=\mathbb{P}\left(P_{\alpha} \leq y\right) .
\end{aligned}
$$

For liminf $\geq$, consider the point process $M$ whose only possible epoch is at $\rho(x)$. The predictable intensity of $M$ is

$$
\lambda(t)=I(\rho(x) \geq t) \beta \bar{B}\left(R_{t-}\right) \geq I(\rho(x)=\infty) \beta \bar{B}\left(d_{x}(t)\right),
$$

and hence

$$
\begin{aligned}
\psi(x, T) & =\mathbb{E} M(T)=\mathbb{E} \int_{0}^{T} \lambda(t) d t \\
& \geq \mathbb{P}(\rho(x)=\infty) \int_{0}^{T} \beta \bar{B}\left(d_{x}(t)\right) d t \\
& =(1-\psi(x)) \int_{x}^{x+\gamma(x) y} \beta \bar{B}(z) \frac{1}{a+b z} d z \\
& =(1-\psi(x))\{G(x+\gamma(x) y)-G(x)\}, \\
\liminf _{x \rightarrow \infty} \frac{\psi(x, T)}{\psi(x)} & \geq \lim _{x \rightarrow \infty} \frac{G(x+\gamma(x) y)-G(x)}{\psi(x)}=\mathbb{P}\left(P_{\alpha} \leq y\right) .
\end{aligned}
$$

The analytic reduction of Lemma 4.1 is carried out in the next section.

\section{Examples.}

5.1. The storage process: more on extremal behavior. Whereas there is no established universal definition of the extremal index $\theta$ in continuous time, we shall take a shortcut and simply adapt the characterization (2.1) as a definition. 
Corollary 5.1. (i) Assume that $r(x) \rightarrow r(\infty), x \rightarrow \infty$, where $\beta \mu_{B}<r(\infty)<$ $\infty$. Then $\theta=0$ and $\max _{t \leq T} V_{t}-\max _{k=1, \ldots, \mid \beta T\rfloor} U_{k} \rightarrow V_{\infty}+M$ in t.v., where $V_{\infty}, M$ are independent and $M$ is distributed as the stationary workload in a $M / G / 1$ queue with arrival intensity $\beta / r(\infty)$ and service time distribution $B$.

(ii) Assume that $r(x) \rightarrow \infty$. Then $\max _{t \leq T} V_{t}-\max _{k=1, \ldots,\lfloor\beta T]} U_{k} \rightarrow V_{\infty}$ in t.v.

PRoOF. Part (i) is shown with minor variants of the reflected random walk case. The only difference in (ii) is that the storage process decreases rapidly after a jump to a large value $x$ so that the probability of exceeding $x$ shortly after the jump [causing the term $W_{\infty}^{(2)}$ in the reflected random walk case and $M$ in (i)] is negligible.

We will see later that in case (ii), the value of $\theta$ depends both on the form of $\bar{B}(x)$ and $r(x)$.

For $A \subseteq[0,1]$, define

$$
N_{T}(A)=\int_{A} I\left(V_{t T}>u_{T}\right) d t
$$

CoROllary 5.2. Assume that $B \in M D A(H)$ with normalizing constants $a_{n}, b_{n}$ and let $u_{T}=x / a_{\lfloor\beta T\rfloor}+b_{\lfloor\beta T\rfloor}$. Then:

(i) if $r(x) \rightarrow r(\infty), \quad x \rightarrow \infty$, where $\beta \mu_{B}<r(\infty)<\infty$, then $(r(\infty)-$ $\left.\beta \mu_{B}\right) N_{T} / \gamma\left(u_{T}\right)$ converges in distribution to a compound Poisson process with intensity $-\log H(x)$ and compounding distribution $\mathbb{P}\left(V_{\alpha} \leq x\right)$;

(ii) if $r(x)=a+b x$, then $\left[b / \log \gamma\left(u_{T}\right)\right] N_{T} \rightarrow_{\mathscr{D}} N$, where $N$ is a Poisson point process with intensity $-\log H(x)$.

The proof is easy and omitted.

5.2. Regularly varying tails. Assume for the rest of this section that $r(x)=a+b x$. Consider first the case $\bar{B}(x) \approx L(x) / x^{\alpha}$ with $L(x)$ slowly varying. Then we have the following corollary, part (i) of which is the main result of [25] (proved there via Laplace transforms).

COROLLARY 5.3. In the regularly varying case:

(i) $\mathbb{P}\left(V_{\infty}>x\right)=\psi(x) \approx \beta L(x) / \alpha b x^{\alpha}$;

(ii) the extremal index is $\theta=\alpha b$;

(iii) the conditional distribution of $\rho(x)$ given $\rho(x)<\infty$ converges to the exponential distribution with rate $\alpha b$. 
Proof. Part (i) is immediate, and (ii) follows then by inserting in (2.1). For (iii), note that, if $T$ and $y$ are connected as in Lemma 4.1, then $y \approx\left(e^{b T}-1\right)(\alpha-1)$. Hence,

$$
\begin{aligned}
\mathbb{P}(\rho(x)>T \mid \rho(x)<\infty) & \rightarrow \mathbb{P}\left(P_{\alpha}>y\right) \\
& =\frac{1}{(1+y /(\alpha-1))^{\alpha}} \approx e^{-\alpha b T} .
\end{aligned}
$$

5.3. Lognormal tails. When $\gamma(x) / x \rightarrow 0$, we have $T \approx \gamma(x) y /(x b)$ in Lemma 4.1, and Lemma 4.1 means that $\delta(x) \rho(x)$ has a limiting standard exponential distribution where $\delta(x)=b x / \gamma(x) \rightarrow \infty$.

Assume in particular that $B$ is lognormal, that is, the distribution of $e^{U}$ where $U$ is standard normal. Then

$$
\begin{aligned}
& \bar{B}(x) \approx \frac{1}{\sqrt{2 \pi} \log x} \exp \left[-(\log x)^{2} / 2\right], \\
& \bar{G}(x) \approx \frac{\beta}{b \sqrt{2 \pi}(\log x)^{2}} \exp \left[-(\log x)^{2} / 2\right], \\
& \gamma(x) \approx \frac{x}{\log x}
\end{aligned}
$$

and we get the following.

COROLLARY 5.4. In the lognormal case:

(i) $\mathbb{P}\left(V_{\infty}>x\right)=\psi(x) \approx\left[\beta / b \sqrt{2 \pi}(\log x)^{2}\right] \exp \left[-(\log x)^{2} / 2\right]$;

(ii) the extremal index is $\theta=\infty$;

(iii) the conditional distribution of $b \log x \rho(x)$ given $\rho(x)<\infty$ is limiting standard exponential.

5.4. Weibull tails. Let now $B$ be DFR (decreasing failure rate) Weibull, $\bar{B}(x)=\exp \left(-x^{\alpha}\right)$ with $0<\alpha<1$. Then

$$
\bar{G}(x) \approx \frac{\beta}{\alpha b x^{\alpha}} \exp \left(-x^{\alpha}\right), \quad \gamma(x) \approx \frac{1}{\alpha} x^{1-\alpha},
$$

and we get the following.

Corollary 5.5. In the DFR Weibull case:

(i) $\mathbb{P}\left(V_{\infty}>x\right)=\psi(x) \approx\left(\beta / \alpha b x^{\alpha}\right) \exp \left(-x^{\alpha}\right)$;

(ii) the extremal index is $\theta=\infty$;

(iii) the conditional distribution of $\alpha b x^{\alpha} \rho(x)$ given $\rho(x)<\infty$ is limiting standard exponential.

It may be noted that the above results for $\psi(x), \psi(x, T)$ are much more complete than what is known for the light-tailed case where the only avail- 
able exact asymptotics is for $\psi(x)$ when $B$ is exponential. We conjecture that when $\bar{B}(x) \approx c x^{\delta} e^{-\eta x}$, then the conditional distribution of $x \rho(x)$ given $\rho(x)<\infty$ is asymptotically exponential.

\section{REFERENCES}

[1] Anantharam, V. (1988). How large delays build up in a GI/GI/1 queue. Queueing Systems Theory Appl. 5 345-368.

[2] Asmussen, S. (1982). Conditioned limit theorems relating a random walk to its associate, with applications to risk reserve processes and the $G I / G / 1$ queue. Adv. in Appl. Probab. 14 143-170.

[3] Asmussen, S. (1987). Applied Probability and Queues. Wiley, New York.

[4] Asmussen, S. (1996). Rare events in the presence of heavy tails. In Stochastic Networks: Rare Events and Stability (P. Glasserman, K. Sigman and D. Yao, eds.) 197-214. Springer, New York.

[5] Asmussen, S. and KlüPPelberg, C. (1995). Large deviations results in the presence of heavy tails, with applications to insurance risk. Stochastic Process. Appl. 64 103-125.

[6] Asmussen, S. and KLÜPPELBERG, C. (1997). Stationary M/G/1 excursions in the presence of heavy tails. J. Appl. Probab. 34 208-212.

[7] Asmussen, S. and Nielsen, H. M. (1995). Ruin probabilities via local adjustment coefficients. J. Appl. Probab. 32 736-755.

[8] Asmussen, S. and Schock Petersen, S. (1989). Ruin probabilities expressed in terms of storage processes. Adv. in Appl. Probab. 20 913-916.

[9] Balkema, A. A. and De HaAn, L. (1974). Residual life-time at great age. Ann. Probab. 2 792-804.

[10] BERman, S. M. (1962). Limit distribution of the maximum term in a sequence of dependent random variables. Ann. Math. Statist. 33 894-908.

[11] Brockwell, P. J., Resnick, S. I. and Tweedie, R. L. (1982). Storage processes with general release rule and additive inputs. Adv. in Appl. Probab. 14 392-433.

[12] Dassios, A. and Embrechts, P. (1989). Martingales and insurance risk. Stochastic Models 5 181-217.

[13] DuRRetT, R. (1980). Conditioned limit theorems for random walks with negative drift. Z. Wahrsch. Verw. Gebiete 52 277-287.

[14] Embrechts, P. and Goldie, C. M. (1980). On closure and factorization properties of subexponential and related distributions. J. Austral. Math. Soc. Ser. A 29 243-256.

[15] Embrechts, P., KlÜPPelberG, C. and Mikosch, T. (1997). Extremal Events in Finance and Insurance. Springer, New York.

[16] Embrechts, P. and Veraverbeke, N. (1982). Estimates for the probability of ruin with special emphasis on the possibility of large claims. Insurance Math. Econom. $155-72$.

[17] Gelub, J. L. and DE HAAN, L. (1987). Regular Variation, Extensions and Tauberian Theorems. CWI Tract 40. CWI, Amsterdam.

[18] Gnedenko, B. V. and Kovalenko, I. N. (1989). Introduction to Queueing Theory, 2nd ed. Birkhäuser, Basel.

[19] Goldie, C. and RESNICK, S. I. (1988). Distributions that are both subexponential and in the domain of attraction of an extreme-value distribution. Adv. in Appl. Probab. 20 706-718.

[20] Harrison, J. M. and Resnick, S. I. (1976). The stationary distribution and first exit probabilities of a storage process with general release rule. Math. Oper. Res. 1 347-358.

[21] HARRison, J. M. and RESNICK, S. I. (1977). The recurrence classification of risk and storage processes. Math. Oper. Res. 3 57-66.

[22] Iglehart, D. L. (1972). Extreme values in the GI/G/1 queue. Ann. Math. Statist. 43 $627-635$.

[23] KeILson, J. (1979). Markov Chain Models—Rarity and Exponentiality. Springer, New York. 
[24] KLÜPPELBERG, C. (1988). Subexponential distributions and integrated tails. J. Appl. Probab. 25 132-141.

[25] KLÜPPElBerg, C. and StadtmülleR, U. (1995). Ruin probabilities in the presence of heavytails and interest rates. Scand. Actuar. J. 49-58.

[26] Leadbetter, M. R., Lindgren, G. and Rootzén, H. (1983). Extremes and Related Properties of Random Sequences and Processes. Springer, New York.

[27] Resnick, S. I. (1987). Extreme Values, Regular Variation Point and Processes. Springer, New York.

[28] Rootzén, H. (1988). Maxima and exceedances of stationary Markov chains. Adv. in Appl. Probab. 20 371-390.

[29] Sundt, B. and Teugels, J. L. (1995). Ruin estimates under interest force. Insurance Math. Econom. 16 7-22.

[30] Sundt, B. and Teugels, J. L. (1996). The adjustment coefficient in ruin estimates under interest force. Insurance Math. Econom.

Department of Mathematical Statistics

UNIVERSITY OF LUND

Box 118

S-221 00 LuND

SWEDEN

E-MAIL: asmus@maths.lth.se 